\title{
Prenatal Programming and Toxicity II (PPTOX II): Role of Environmental Stressors in the Developmental Origins of Disease
}

It has been over three years since Reproductive Toxicology produced a special issue: Fetal Basis of Adult Disease. ${ }^{1}$ That issue contained 22 articles which highlighted this emerging field. Now the field has matured significantly and to highlight the advances an international conference, PPTOX II, was held in Miami Florida, December 7-10 2009. This conference followed PPTOX I which was held in the Faroe Islands in 2007. Highlights of that meeting were published in Basic \& Clinical Pharmacology \& Toxicology. ${ }^{2}$

The focus of the PPTOX II meeting was on the fact that fetal and early postnatal development constitutes the most vulnerable time period of human life, with regard to adverse effects of environmental hazards. Subtle effects during development can lead to functional deficits and increased disease risk later in life. Indeed there are now experimental data for the role of developmental exposures to environmental chemicals for male and female reproductive diseases/dysfunctions, prostate, breast and uterine cancers, cardiopulmonary diseases/dysfunctions, neurobehavioral problems, and neurodegenerative diseases and immune and autoimmune diseases. Exposure to environmental chemicals during prenatal and early postnatal environment affects gene expression and the resulting epigenetic changes may constitute an important mechanism for the programming effects. Since the epigenetic system is most vulnerable to disruption during specific times during development, timing of environmental exposures is critical for their effects on disease etiology. It is also apparent from animal studies that epigenetic changes due to environmental exposures during development can be used as biomarkers of both exposure and potential future disease or dysfunction.

The objective of the conference was to examine the animal and human data supporting the role of environmental exposures in the developmental origins of disease hypothesis. The meeting was organized by disease in order to review the current state of the literature and to identify mechanisms for the effects of environmental agents on each disease as well as to identify research gaps and challenges and to integrate the basic and applied science. Because of its International nature, the multidisciplinary and interdisciplinary nature of the speakers, and the attendance and participation of students from multiple disciplines, a major goal was to stimulate collaborations that will lead to the development and validation of biomarkers of exposure and disease susceptibility, improvement of exposure assessment and extrapolation across species and routes of exposure.

The four-day conference was sponsored by the Society of Toxicology, the National Institute of Environmental Health Sciences, World Health Organization, U.S. Environmental Protection Agency, the Centers for Disease Control and Prevention, the Eunice Kennedy Shriver National Institute of Child Health and Human Development, the European Environment Agency, the National Center for Toxicological
Research/FDA, the Superfund Research Program, the National Cancer Institute, National Institute for Environmental Studies, the International Union of Toxicology and the Agency for Toxic Substances and Disease Registry.

We hope this special issue of Journal of Developmental Origins of Health and Disease will stimulate continued research into this important area that links environmental exposures during development to diseases later in life. This research paradigm has the potential to actually reduce disease incidence by reducing exposures in utero and early in life and by developing biomarkers that predict future disease onset and then developing interventions to prevent disease etiology.

Details of the meeting including the program can be found online ${ }^{3}$ as well as a link to the first PPTOX meeting. ${ }^{4}$

Sally Darney, Ph.D.

National Program Director for Human Health Office of Reseach and Development U.S. Environmental Protection Agency

Bruce Fowler, Ph.D.

Assistant Director for Science

Division of Toxicology and Environmental Medicine, ATSDR Centers for Disease Control

Philippe Grandjean, M.D. Professor, Head of Research University of Southern Denmark

Jerrold Heindel, Ph.D. Chief Cellular, Organs and Systems Pathobiology Branch National Institute of Environmental Health Sciences

Donald Mattison, M.D. Senior Advisor to the Director of NICHD

Eunice Kennedy Shriver National Institute of Child Health and

Human Development

National Institutes of Health, HHS

William Slikker, Jr., Ph.D. Director, National Center for Toxicological Research U.S. Food and Drug Administration

\section{References}

1. Reproductive Toxicology, Volume 23, Issue 3, Pages 257-470 (April-May 2007).

2. Basic \& Clinical Pharmacology \& Toxicology, Volume 102, Issue 2, February 2008.

3. https://www.toxicology.org/ai/meet/cct_pptox_meeting.asp (Accessed 10th February 2011).

4. http://www.pptox.dk (Accessed 10th February 2011). 